\title{
In Situ XPS Reveals Voltage Driven Asymmetric lon Movement of an Ionic Liquid through the Pores of a Multilayer Graphene Electrode
}

\author{
Merve Taner Camci, ${ }^{\dagger, \S}$ Burak Ulgut, ${ }^{\dagger}$ Coskun Kocabas, ${ }^{\ddagger} \|_{\odot}$ and Sefik Suzer ${ }^{* \dagger}{ }^{\dagger}$ \\ ${ }^{\dagger}$ Department of Chemistry and ${ }^{\ddagger}$ Department of Physics, Bilkent University, 06800 Ankara, Turkey
}

\begin{abstract}
Under application of a voltage bias, asymmetric ion movement of an ionic liquid (IL) through a multilayered graphene (MLG) electrode has been detected by X-ray photoelectron spectroscopy, via recording the intensity of the two nitrogen peaks. Accordingly, we observe that upon increasing the bias, the two peaks representing the cationic and anionic fragments of the IL start appearing with increasing intensity, together with an asymmetry in their ratio, differing from unity by about $10 \%$. Bias-dependent binding energy shifts followed through atomic features of the IL (F 1s, N $1 \mathrm{~s}$, and $\mathrm{C} 1 \mathrm{~s})$ and the graphene electrode (C 1s) indicate that a distinct solid-liquid interface develops throughout the entire intercalation process with an additional and pertinent evidence for finite potential drops across the two electrical double layers. This evidence is bolstered by the fact that the measured binding energy difference between the F 1s of the liquid and C 1s peak of the semisolid MLG electrode is only about half of the applied bias, where the rest of the applied voltage is screened by the two electrical double layers at the solid-liquid interfaces between (i) the MLG-IL and (ii) IL-bottom metal electrode. A simple electrostatic estimation indicates that even this small $10 \%$ ion imbalance would lead to 4 orders of magnitude larger voltage development between the IL and the MLG phases and suggests the need for amendment(s) to the current understanding of the dielectric description of ILs.
\end{abstract}

\section{INTRODUCTION}

Charge distribution, storage, and movement in solutions and soft matter are of paramount importance for understanding and rational use of various electrochemical concepts toward advanced applications in energy and chemical, biochemical, microfluidics, and sensing applications. Over the last 3 decades, the vast research efforts using advanced measurement and computational and simulation techniques have changed the concept of electroneutrality in many physicochemical processes taking place in solution surfaces or in pores of solid materials. ${ }^{1}$

Being a chemically sensitive and quantitative surface analysis technique, X-ray photoelectron spectroscopy (XPS) has been the pivotal method to extract information about distribution of cations and anions on various surface structures once difficulties in handling liquids had been overcome through multiple advancements in experimental techniques. The pioneering XPS investigation of ion enrichment on surfaces of viscous liquids by H. Siegbahn ${ }^{2-5}$ was later successfully extended to aqueous solutions in the form of continuous jets using synchrotronbased photoemission spectroscopy by Faubel and co-workers. ${ }^{6}$ In parallel, developments in ambient pressure XPS have enabled investigation of a large variety of critical materials and chemical processes. ${ }^{7,8}$ All of these giant leaps and developments have also been successfully supported and guided by extensive molecular dynamics simulations., 9

Another important scientific advancement has emerged from a completely different focus area, investigation of porous materials by NMR, another quantitative spectroscopic technique for investigation of electrochemical energy generation and storage systems. ${ }^{11-16}$ In all of these studies, it was explicitly shown that the anion/cation ratio strongly deviated from stoichiometry, depending on their chemical nature (i.e., specific ion effects) and was also controlled by the polarity of the applied electrical fields. However, although implied, local breakdown of the electroneutrality had not been mentioned explicitly, except in the work of Luo et al. ${ }^{13}$ Different aspects of these experimental and theoretical developments have recently been reviewed. ${ }^{17-19}$

Nonvolatile room-temperature ionic liquid (IL) electrolytes have allowed us and others to utilize lab-based XPS instruments for investigating various electrochemical processes under ultrahigh vacuum conditions, without the need for extensive pumping techniques or synchrotron facilities. ${ }^{20-30}$ In one of our recent studies, we have used a multilayered graphene (MLG) electrode on paper to fabricate an optically reconfigurable device using an ionic liquid as the electrolyte, ${ }^{31}$ which was also adopted for terahertz radiation ${ }^{32}$ or flexible electrochromic devices ${ }^{33}$ and tunable fractal metasurfaces. ${ }^{34}$ In this contribution, we report on using a similar multilayered graphene as the top electrode and utilize XPS to monitor in situ (i) the changes in the anion/cation intensity ratio under applied electric fields and (ii) the electrical potential developments on different surface structures, which are derived from the shifts in the binding energies of the corresponding atomic core levels in a chemically resolved fashion. ${ }^{35,36}$

\section{EXPERIMENTAL SECTION}

A porous polyethylene membrane (PEM) separator is used to allow the IL to pass but prevent shorting of the top MLG and

Received: March 22, 2018

Revised: $\quad$ May 3, 2018

Published: May 17, 2018 

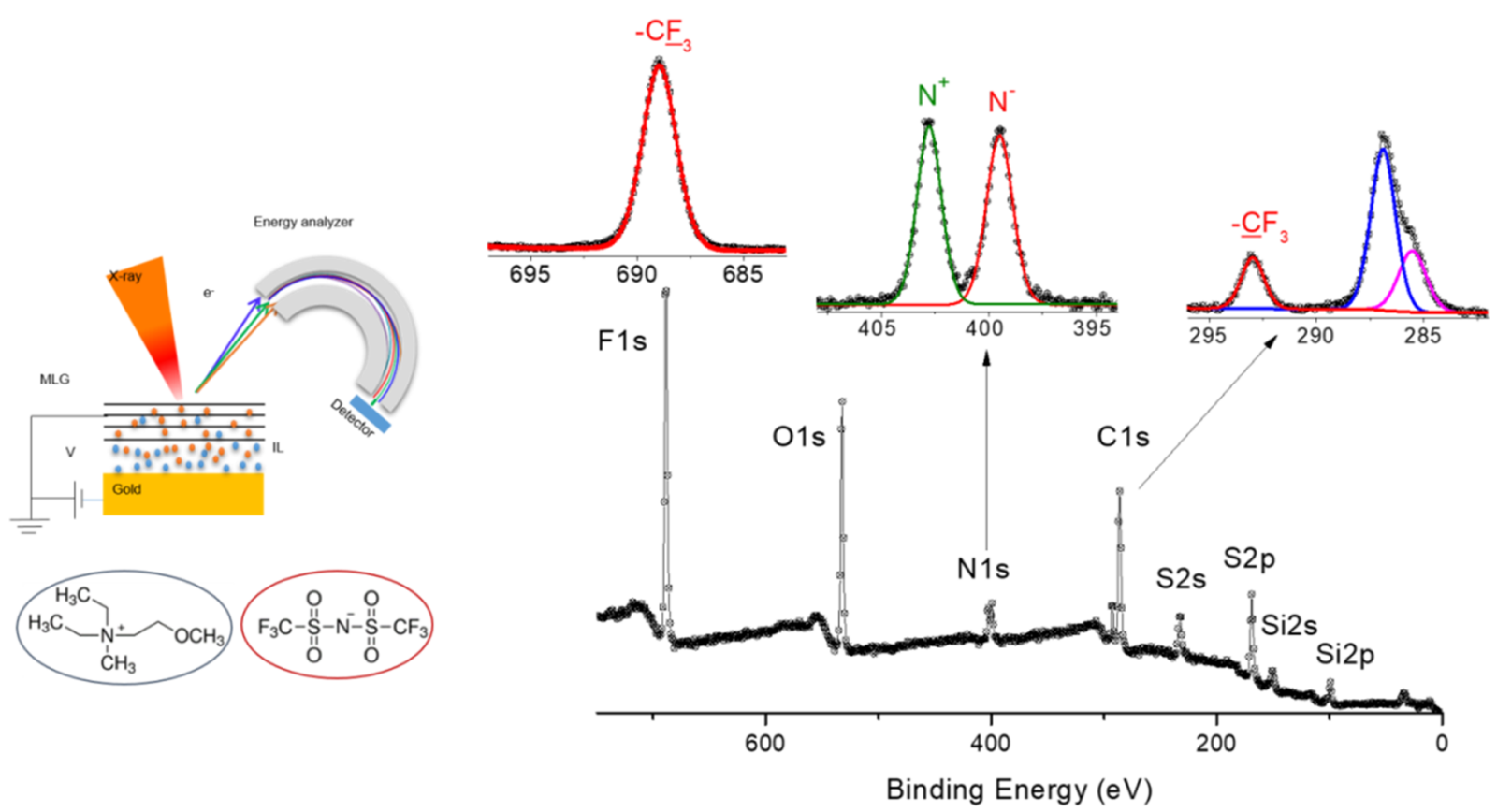

Figure 1. Experimental setup used for the operando-XPS in the back-gate geometry. Chemical structure of the ionic liquid and its survey and region's XP spectra.
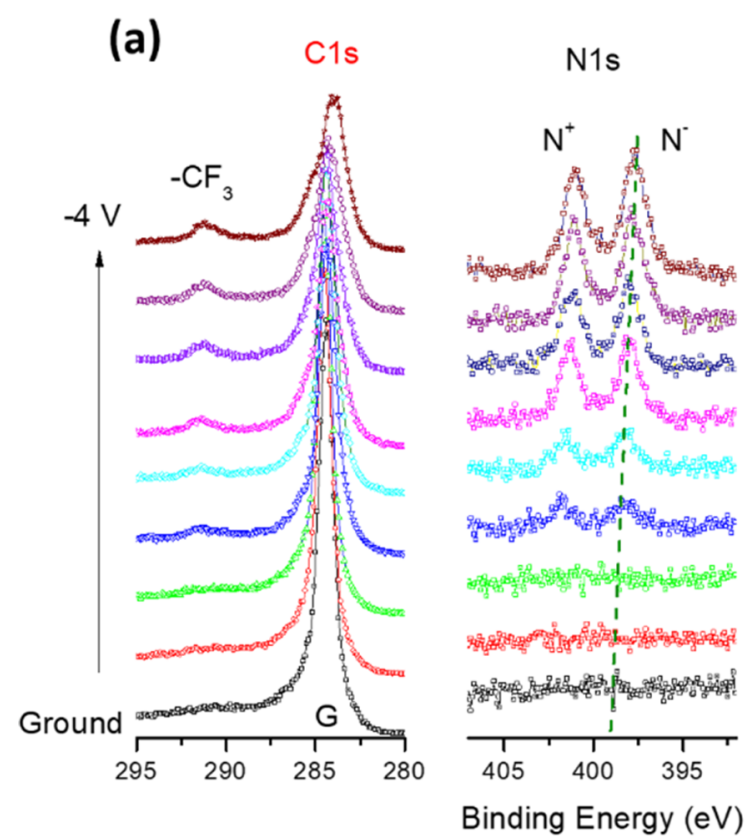

(b)

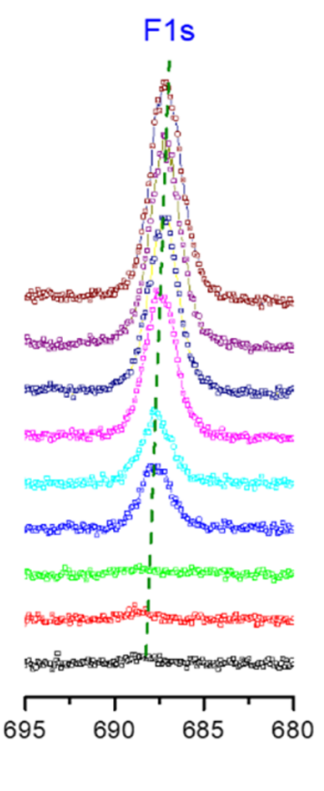

(c)
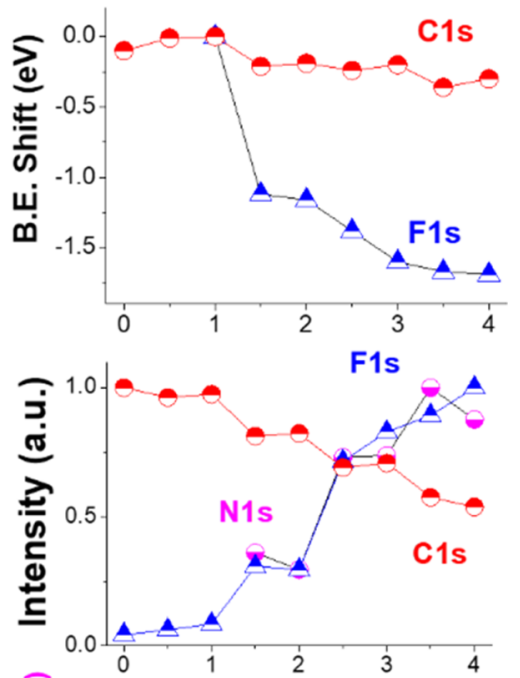

(d)

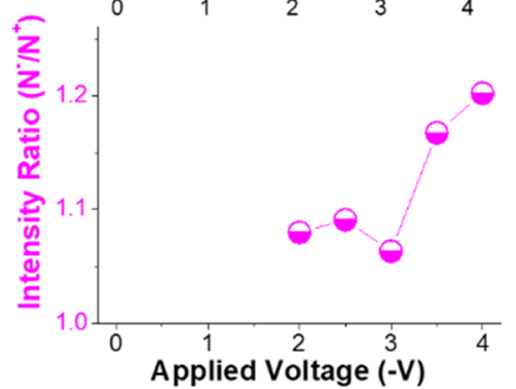

Figure 2. (a) XP spectra of the surface of the device under bias voltages changing from 0 to $-4 \mathrm{~V}$. (b) Normalized intensities of $\mathrm{C}$ 1s, $\mathrm{N}$ 1s, and $\mathrm{F} 1 \mathrm{~s}$. (c) Variation in the $\mathrm{N}^{-} / \mathrm{N}^{+}$intensity ratio. (d) Variation in the binding energy of $\mathrm{C}$ 1s, representing the MLG and F $1 \mathrm{~s}$ and $\mathrm{N} 1 \mathrm{~s}$ representing the IL.

bottom gold electrodes. Multilayer graphene samples are grown on nickel foils (Alfa Aesar) using chemical vapor deposition at temperatures ranging from 850 to $1000{ }^{\circ} \mathrm{C}$ at ambient pressure. A mixture of $\mathrm{H}_{2}, \mathrm{Ar}$, and $\mathrm{CH}_{4}$ gases is used during the growth, with flow rates set as 100,100, and $30 \mathrm{sccm}$, respectively, and the growth time is $5 \mathrm{~min}$. This procedure enables fabricating multilayer graphene samples having 100-250 layers. ${ }^{33}$ The MLG electrode is transferred onto the PEM, which is directly placed on the gold electrode, and the ionic liquid is initially introduced in between the PEM and the bottom electrode. A Thermo Fisher K-Alpha X-ray photoelectron spectrometer with a monochromatized photon energy of $1486.6 \mathrm{eV}$ has been used 

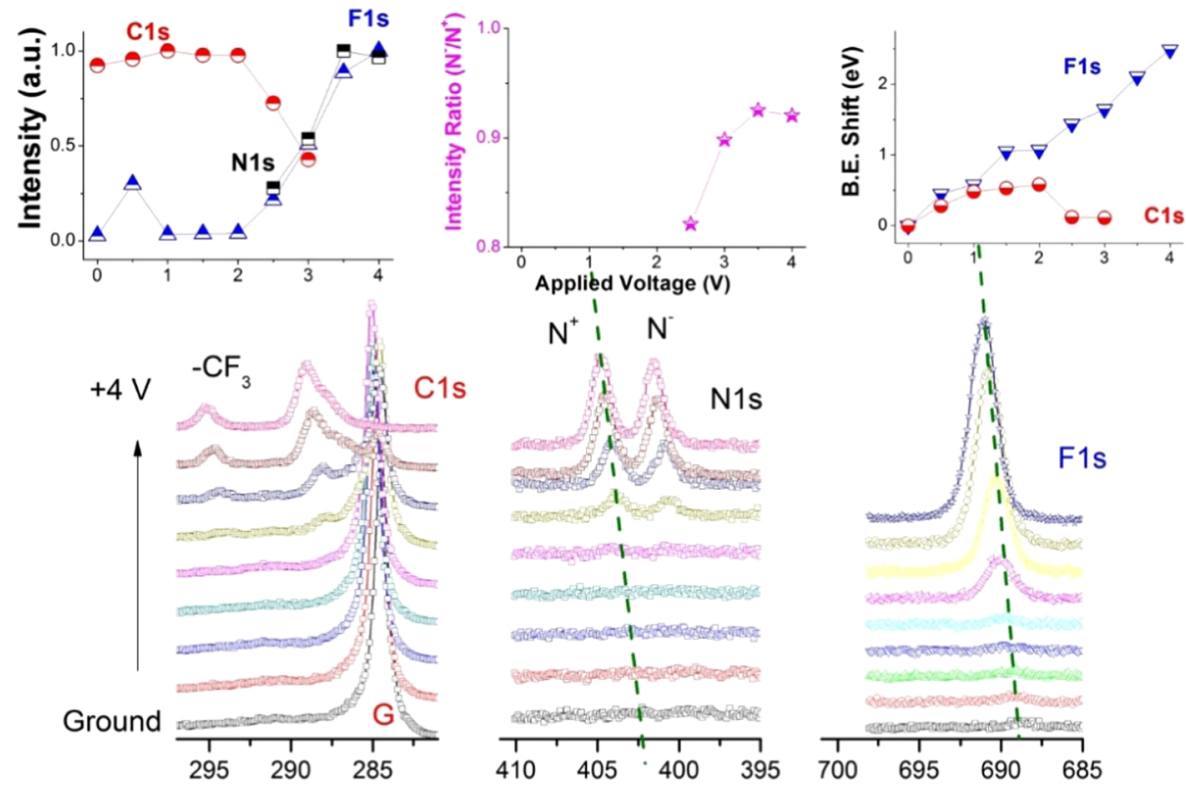

Figure 3. XP spectra recorded from the surface of the device under bias voltages changing from 0 to $+4 \mathrm{~V}$.

to collect data for all measurements. Direct current external bias is applied to either the MLG or the bottom electrode using a Keithley 2400 source-meter electrical signal. Data processing is carried out using the Avantage software package provided by the manufacturer.

\section{RESULTS AND DISCUSSION}

The ionic liquid used, [N,N-diethyl-N-methyl- $N$ - $(2-$ methoxyethyl)ammonium bis(trifluoromethanesulfonyl)imide] DEME-TFSI, has two nitrogen moieties, one representing the cation and the other the anion. The corresponding $\mathrm{N}$ 1s core levels are conveniently separated in the energy scale by $3.2 \mathrm{eV}$. We also utilize the unique property of XPS to monitor the electrical potentials developed locally since the relevant data are derived from the measured kinetic (or binding) energy of the emitted photoelectrons, which are directly affected by the developed or externally applied electrical fields. ${ }^{29,30}$ XPS has long been successfully used to monitor various in situ processes, such as oxidation, reduction, ${ }^{21-24,37-41}$ intercalation, ${ }^{42-48}$ overpotential developments, ${ }^{49}$ etc. In this work, we report a direct observation of a stoichiometric imbalance or asymmetric ion movement by XPS, complemented with a potential drop measurement, under the influence of an applied electric field.

Deviations from the stoichiometry or charge imbalances in surface structures of materials containing ionic liquids by XPS have long been reported and discussed, mostly in terms of chemical compositions, interactions, or reactions. ${ }^{22,23,27,40,49-53}$ Our strategy is different since we monitor the changes under working conditions (in Operando) in electrochemical device(s). Figure 1 shows the experimental setup and the schematics of the device together with a survey spectrum of the neat $\mathrm{IL}$, where the stoichiometric cation/anion ratio is spectroscopically validated by the two equally intense $\mathrm{N}$ 1s peaks representing the cation and anion, respectively.

In Figure 2, we show the spectral evolution of the various components, under increasing imposed bias potentials $\left(\Phi_{\mathrm{B}}\right)$. At the beginning, only the $\mathrm{C} 1 \mathrm{~s}$ peak of the graphene is observed as the major component, with the $\mathrm{F} 1 \mathrm{~s}$ as a very weak one, most probably due to contamination. Starting from $-1.5 \mathrm{~V}$ and onward, peaks representative of the IL become observable with a continuous increase in their intensity, till the start of electrochemical decomposition of the IL around $-4.0 \mathrm{~V}$, easily noticeable with an increase in the spectrometer background pressure. These spectral evolutions provide a wealth of information about the operation of the device and composition of the analyzed regions.

Appearance of $\mathrm{N} 1 \mathrm{~s}$ and $\mathrm{F} 1 \mathrm{~s}$ peaks after $1.5 \mathrm{~V}$ indicates the onset of the intercalation process and the threshold voltage. This onset voltage is comparable to the same value determined using both electrochemical and XPS measurements for perchlorate ion intercalation in highly oriented pyrolytic graphite from aqueous solutions. ${ }^{46,47}$ Since XPS probes the very top surface $(\sim 5-6 \mathrm{~nm})$, appearance of $F 1$ s and $N$ is peaks shows that the ions can efficiently intercalate the thick active surface ( $>100$ graphene layers). The intensity of $C 1 s$ decreases with increasing voltage due to the partial coverage of the top surface with the IL. The $\mathrm{C} 1 \mathrm{~s}$ peak of the $-\mathrm{CF}_{3}$ group associated with IL also appears after the threshold voltage.

Although the graphene surface is grounded, the binding energy of $\mathrm{C} 1 \mathrm{~s}$ also experiences small shifts with the applied bias, from 284.37 to 283.67 , most likely due to the shift in the Fermi energy of graphene. ${ }^{54}$ Interestingly, we also observe cointercalation of anions and cations of the ionic liquid with a measurable ion/charge imbalance ( $>10 \%$ change in the ratio of $\mathrm{N}^{-}$to $\mathrm{N}^{+}$). This charge imbalance (due to mobile and quasiindependent ions) is also responsible for electrostatic doping on graphene layers. When we apply positive bias voltage, the charge imbalance is reversed (Figure 3).

Implicit in our arguments is a picture of the MLG electrode as a loose but conducting network of carbon atoms electrically connected to each other and to the grounding wire and the ionic liquid as the second but less conducting network of electrolyte medium positioned between the two electrodes. The grounding wire brings the Fermi level of the top electrode to that of the spectrometer, but the Fermi level of the bottom electrode is shifted with the applied bias potential $\left(\Phi_{\mathrm{B}}\right){ }^{55-57}$ As a result, if we were able to measure the kinetic energy of any core level representative of the bottom electrode (not possible due to the limited XPS probe depth, $<6 \mathrm{~nm}$ ), a complying shift 

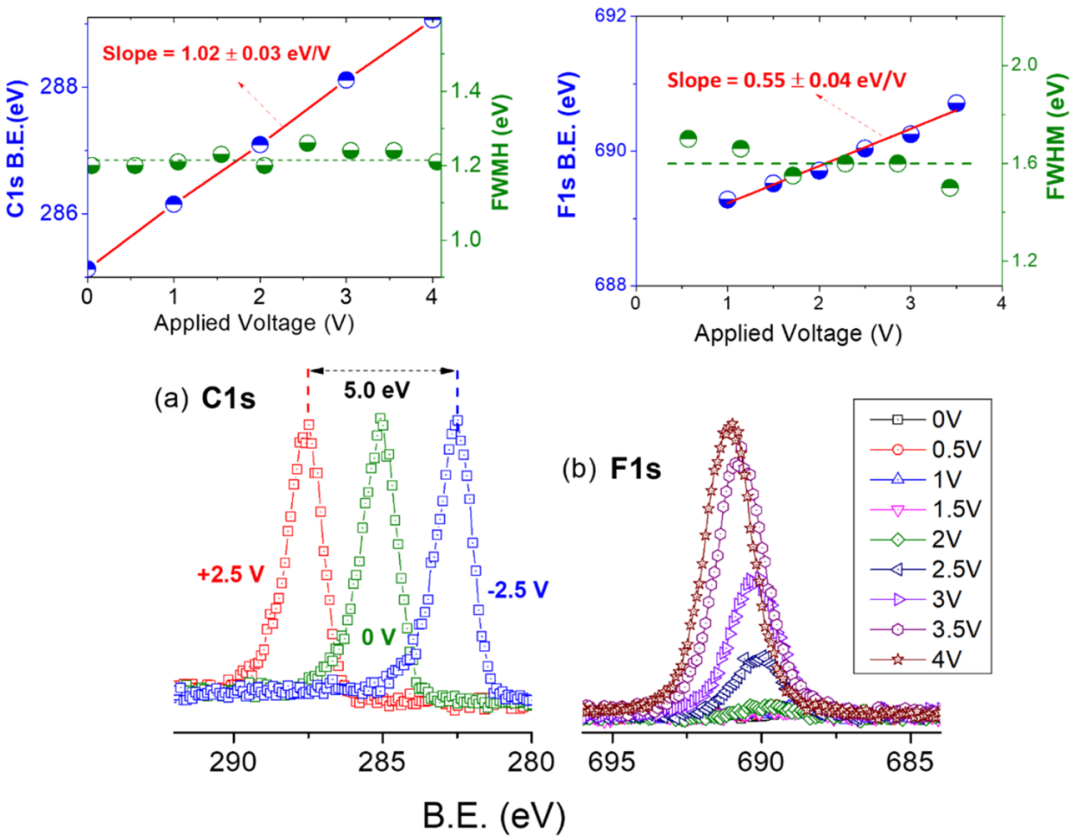

Figure 4. (a) $\mathrm{C} 1 \mathrm{~s}$ region of a few-layer graphene on $\mathrm{Cu}$, recorded under $+2.5,0$, and $-2.5 \mathrm{~V}$ bias, where the shifts in the binding energy positions comply exactly with the applied bias. (b) Details of the F 1 s region recorded from the surface of the device under bias, changing from 0 to $+4 \mathrm{~V}$ (same as those in Figure 2). Insets show linear fits (red line) of the position (blue dots) and the full-width half-maximum of the C 1s (graphene on $\mathrm{Cu}$ ) and F 1s (IL on MLG electrode) peaks (green dots) against the applied bias. For the linear fit of F 1s, points at $0 \mathrm{~V}$ (surface impurity) and $4 \mathrm{~V}$ (decomposition) are excluded.

C1s N1s

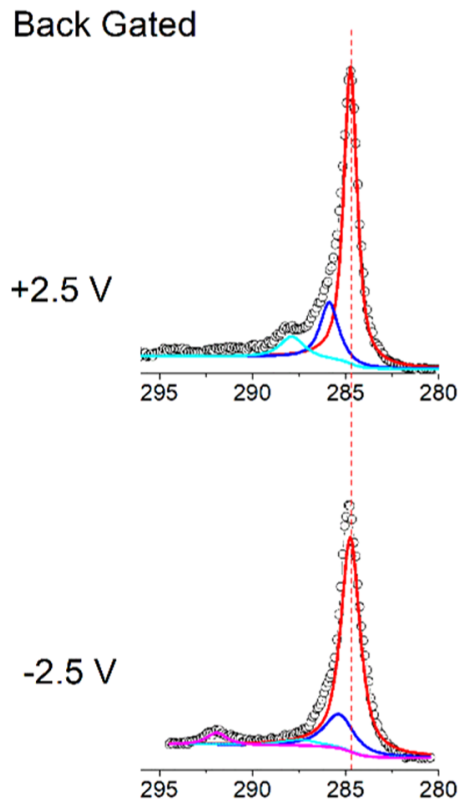

F1s

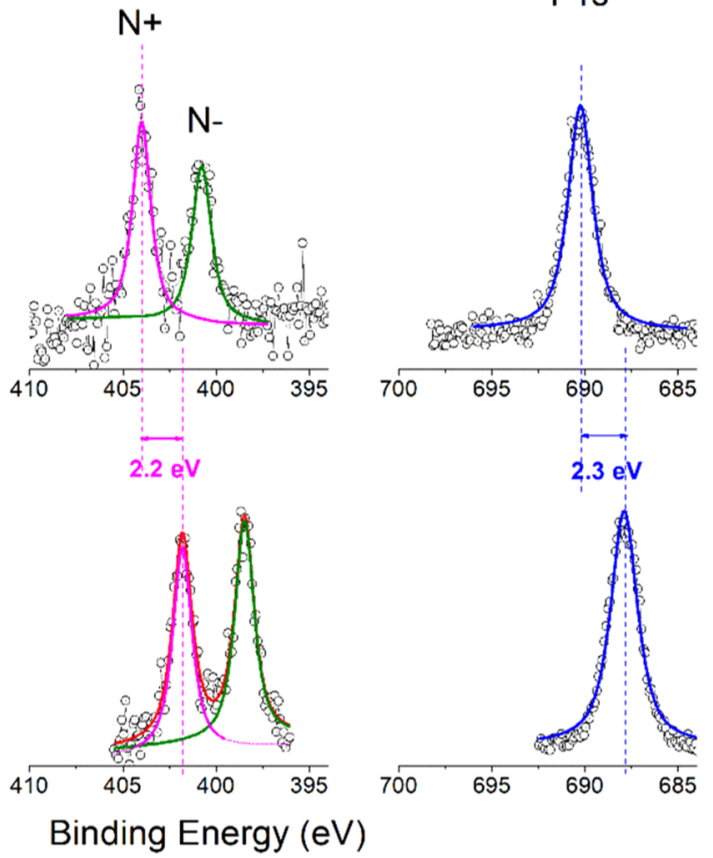

Figure 5. C 1s, F 1s, and N 1s regions of the intercalated thick graphene layer with the IL at +2.5 and $-2.5 \mathrm{~V}$ back-gate voltages.

of $-e \Phi_{\mathrm{B}}$ (in $\mathrm{eV}$ ) would have been measured, where $e$ is the electron's charge. A negative bias accelerates the outgoing photoelectrons, resulting in an apparent decrease $(\mathrm{BE}=\hbar \nu-$ $\mathrm{KE}$ ) in the measured binding energy position, and the positive bias works in the opposite direction, as shown in Figure 4a for a few-layer graphene grown on $\mathrm{Cu}$. On the other hand, peaks belonging to the IL moieties ( $\mathrm{F} 1 \mathrm{~s}, \mathrm{~N} 1 \mathrm{~s}$, and $\mathrm{C} 1 \mathrm{~s}$ ), intercalated in the grounded graphene layers, but also in electrical contact with the bottom Au electrode, display shifts with the bias but not fully complying with it $\left(-e \Phi_{\mathrm{B} \text {,electrolyte }}\right)$, as depicted in Figure $4 \mathrm{~b}$ by the recorded $\mathrm{F}$ 1s peaks under backgated positive bias.

A linear fit to the data of Figure $4 \mathrm{~b}$ gives a slope of $0.55 \pm$ $0.04 \mathrm{eV} / \mathrm{V}$, very close to half of the applied bias, which we assign to the electrical potential of the liquid medium. Furthermore, and as also shown in the same figure, no 
additional broadening of the peaks is observed, attesting to the uniformity of the potentials developed on the liquid surface. ${ }^{36}$ As discussed in detail in our previous work, ${ }^{29}$ this finding reflects directly the efficient screening through the formation of two electrical double layers (EDLs) at both solid-liquid interfaces. $^{54,55}$ Entirely similar findings were also obtained when XPS data was recorded under application of the bias through the top MLG electrode, reflecting the consistency of our measurements.

As a further proof of the observed noncomplying shifts of the liquid phase, we show in Figure 5 the difference in the $\mathrm{N}$ 1s and $\mathrm{F}$ 1s peaks under positive and negative $2.5 \mathrm{~V}$ biases, which are measured as 2.2 and $2.3 \mathrm{eV}$, slightly less than half of the bias difference of $5.0 \mathrm{eV}$ [see Figure 4a], between the MLG-IL and IL-Au-bottom electrodes. The voltage drops on the two EDLs are slightly unequal (the average of the two measured shifts, $2.25 \mathrm{eV}$ and $5.0-2.25=2.75 \mathrm{eV}$ ) due to the different capacitances of the two EDLs and reflect that the MLG-IL interface has a slightly larger capacitance $(2.75 / 2.25=1.2)$ compared to that of the IL-Au electrode due most probably to the larger and fractal contact area between the MLG electrode and the IL, as depicted in Scheme 1.

Scheme 1. Schematics of Ion and Potential Distributions under $-2.5 \mathrm{~V}$ Back Gating

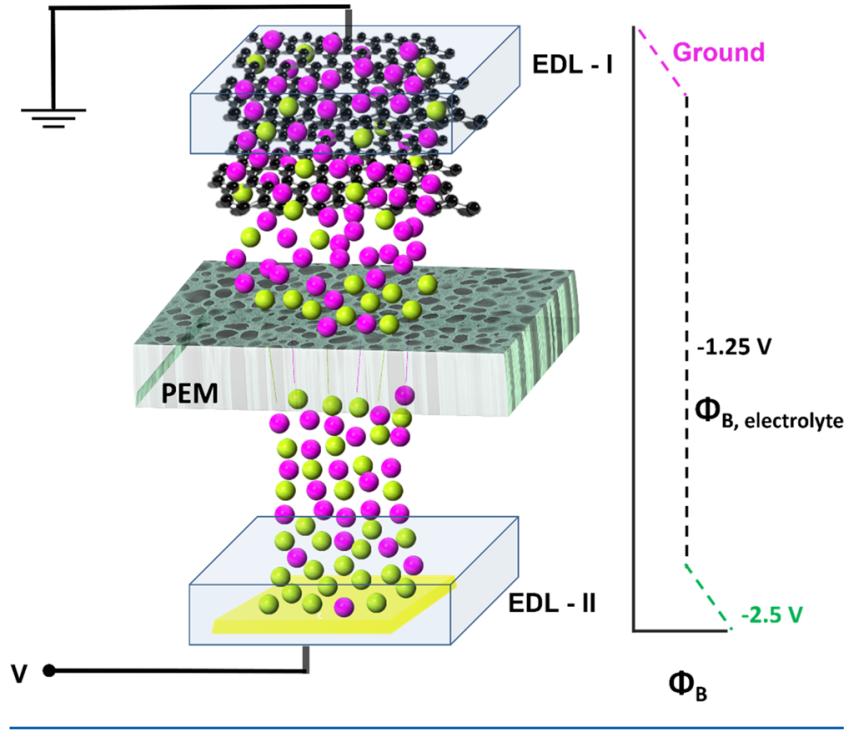

The quantitative nature of XPS enables us to also obtain an order of magnitude estimation of the extent of the intercalation of the ionic liquid in the multilayer graphene at any potential applied. For example, using the intensities of the $\mathrm{F} 1 \mathrm{~s}$ and $\mathrm{C} 1 \mathrm{~s}$ at $+2.5 \mathrm{~V}$, we get an atomic ratio of $\mathrm{F} / \mathrm{C}=0.053$. Taking into account the chemical formula of the $\mathrm{IL}\left(\mathrm{C}_{10} \mathrm{~F}_{6}\right)$, one gets approximately one ion pair for $\sim 110 \mathrm{C}$ atoms of the graphene. Hence, the $\sim 10 \%$ excess positive charge derived from the nitrogen intensity corresponds to one excess positive charge for every $\sim 1100 \mathrm{C}$ atoms of the graphene layers. Using approximately a circular X-ray spot size of $200 \mu \mathrm{m}$ and assuming the probe depth of $6 \mathrm{~nm}$, the analyzed volume can be estimated as $\left[4 \pi \times(200 \mu \mathrm{m})^{2} \times 6 \mathrm{~nm}\right]=3 \times 10^{-9} \mathrm{~cm}^{3}$. Assuming further that density of the MLG layer is close to that of graphite $\left(2 \mathrm{~g} / \mathrm{cm}^{3}\right)$, we can estimate that the analyzed volume contains approximately $5 \times 10^{-8} \mathrm{~mol}$ of carbon atoms to yield $10^{-12} \mathrm{~mol}$ or $3 \times 10^{11}$ excess ions and a surface charge density (SCD) of $0.2 \mathrm{C} / \mathrm{m}^{2}$. This number is in the same order of magnitude as that of the SCD derived from the surface potentials measured using XPS of aqueous silica colloidal nanoparticles charged upon changing the $\mathrm{pH}$ of the solution from 0.3 to $10.0 .^{56,57}$ However, the same SCD is at odds with the estimation of the electrical potential development on the layer, as shown below. Since the device geometry adopted is similar to that of a parallel plate capacitor of a thickness $d(\sim 1$ $\mathrm{mm}$ ), containing mostly the IL as the dielectric (with a dielectric constant of $\varepsilon \sim 20),{ }^{58}$ the electrical potential $\Phi$ can be obtained as

$$
\begin{aligned}
\Phi & =\frac{q}{4 \pi \varepsilon \varepsilon_{0} d}=\frac{3 \times 10^{11} \times 1.6 \times 10^{-19} \mathrm{C}}{4 \pi \times 20 \times 8.85 \times 10^{-12} \frac{\mathrm{F}}{\mathrm{m}} \times 10^{-3} \mathrm{~m}} \\
& \approx 2 \times 10^{4} \mathrm{~V}
\end{aligned}
$$

This simple order of magnitude estimation gives an unreasonably large potential. As discussed above, the potential difference developed between the IL medium and the graphene layers (near ground) is measured as $\sim 1.2 \mathrm{~V}$ due to the doublelayer screening ${ }^{29}$ and is 4 orders of magnitude lower than the estimated one. Although the $10 \%$ extra charge we are reporting is in agreement with the findings of MacFarlane et al. through conductivity/viscosity correlations, ${ }^{59}$ for the same number to yield a reasonable voltage on the order of few volts, this simple dielectric picture needs to be amended. Either the local dielectric constant is much higher or there are other effects changing the understanding of the system. Ionicity and overcrowding have been heavily discussed in the recent literature, which can be contributing factors to the low voltage that is induced by a high charge excess. ${ }^{17}$ In that, the structure is such that most of the excess ions are screened by the rest and do not induce extra voltage. This violates the assumption made in the above order of magnitude estimate where excess charges are homogeneously distributed within the volume.

\section{CONCLUSIONS}

In summary, two important assets of XPS have been utilized: (i) quantification of the nonstoichiometric ion movement/ intercalation through the pores of a multilayer graphene top electrode in response to voltage bias and (ii) mapping of the electrical potential developments on the graphene electrode intercalated with the IL, in a totally noninvasive and chemically specific fashion. Although only the potential difference between the grounded top graphene electrode and the surface IL moieties is measured, as functions of the voltage bias, the outcome, guided also by our earlier findings, is claimed to reflect the voltage drops across the two electrical double layers between the ionic liquid and the top and bottom electrodes. Furthermore, using the measured ion enrichment of about $10 \%$, it is estimated that the electrical potential developed would be 4 orders of magnitude larger than the measured value of $\sim 1.2 \mathrm{~V}$, which casts doubts on the traditional approach of treating ILs as simple dielectric media. All of these findings are new and are expected to impact both the fundamental understanding of certain electrochemical concepts and more importantly better usage of electrode/electrolyte systems for applications in energy harvesting and storage, since the geometry of the device used in this work has a close similarity, in both chemical makeup and geometry, to the electrical double-layered supercapacitors. 


\section{AUTHOR INFORMATION}

\section{Corresponding Author}

*E-mail: suzer@fen.bilkent.edu.tr.

ORCID $\odot$

Burak Ulgut: 0000-0002-4402-0033

Coskun Kocabas: 0000-0003-0831-5552

Sefik Suzer: 0000-0002-5866-2600

Present Addresses

${ }^{\S}$ CNRS Institut Charles Sadron, Strasbourg 67034, France (M.T.C.).

"School of Materials, The University of Manchester, Manchester M13 9PL, U.K. (C.K.).

\section{Author Contributions}

The manuscript was written through contributions of all authors. All authors have given approval to the final version of the manuscript. All authors contributed equally.

Notes

The authors declare no competing financial interest.

\section{ACKNOWLEDGMENTS}

This work was partially supported by the Scientific and Technological Research Council of Turkey (TUBITAK), grant no. 215Z534; C.K. also acknowledges the support from the European Research Council (ERC) Consolidator grant ERC no. 682723 Smart Graphene.

\section{ABBREVIATIONS}

DEME-TFSI, $N, N$-diethyl- $N$-methyl- $N$-(2-methoxyethyl)ammonium bis(trifluoromethanesulfonyl)imide; EDL, electrical double layer; RTIL, room-temperature ionic liquid; MLG, multilayered graphene; SCD, surface charge density; XPS, X-ray photoelectron spectroscopy

\section{REFERENCES}

(1) Dickinson, E. J.; Limon-Petersen, J. G.; Compton, R. G. The Electroneutrality Approximation in Electrochemistry. J. Solid State Electrochem. 2011, 15, 1335-1345.

(2) Siegbahn, M.; Lundholm, M. A Method of Depressing GaseousPhase Electron Lines in Liquid-Phase ESCA Spectra. J. Electron Spectrosc. Relat. Phenom. 1982, 28, 135.

(3) Siegbahn, H. Electron Spectroscopy for Chemical Analysis of Liquids and Solutions. J. Phys. Chem. 1985, 89, 897-909.

(4) Moberg, R.; Bökman, F.; Bohman, O.; Siegbahn, H. ESCA Studies of Phase-Transfer Catalysts in Solution: Ion Pairing and Surface Activity. J. Am. Chem. Soc. 1991, 113, 3663-3667.

(5) Boekman, F.; Bohman, O.; Siegbahn, H. ESCA Studies of Phase Transfer Catalysts in Solution. 2. Surface Ion Pairing and Salting-Out Effects. J. Phys. Chem. 1992, 96, 2278-2283.

(6) Winter, B.; Faubel, M. Photoemission from Liquid Aqueous Solutions. Chem. Rev. 2006, 106, 1176-1211.

(7) Ghosal, S.; Hemminger, J. C.; Bluhm, H.; Mun, B. S.; Hebenstreit, E. L.; Ketteler, G.; Ogletree, D. F.; Requejo, F. G.; Salmeron, M. Electron Spectroscopy of Aqueous Solution Interfaces Reveals Surface Enhancement of Halides. Science 2005, 307, 563-566.

(8) Bluhm, H.; Andersson, K.; Araki, T.; Benzerara, K.; Brown, G. E.; Dynes, J. J.; Ghosal, S.; Gilles, M. K.; Hansen, H.-C.; Hemminger, J. Soft X-ray Microscopy and Spectroscopy at the Molecular Environmental Science Beamline at the Advanced Light Source. J. Electron Spectrosc. Relat. Phenom. 2006, 150, 86-104.

(9) Jungwirth, P.; Tobias, D. J. Specific Ion Effects at the Air/Water Interface. Chem. Rev. 2006, 106, 1259-1281.

(10) Tobias, D. J.; Hemminger, J. C. Getting Specific About Specific Ion Effects. Science 2008, 319, 1197-1198.
(11) Wang, H.; Forse, A. C.; Griffin, J. M.; Trease, N. M.; Trognko, L.; Taberna, P.-L.; Simon, P.; Grey, C. P. In situ NMR Spectroscopy of Supercapacitors: Insight into the Charge Storage Mechanism. J. Am. Chem. Soc. 2013, 135, 18968-18980.

(12) Forse, A. C.; Griffin, J. M.; Merlet, C.; Bayley, P. M.; Wang, H.; Simon, P.; Grey, C. P. NMR Study of Ion Dynamics and Charge Storage in Ionic Liquid Supercapacitors. J. Am. Chem. Soc. 2015, 137, $7231-7242$

(13) Luo, Z.-X.; Xing, Y.-Z.; Ling, Y.-C.; Kleinhammes, A.; Wu, Y. Electroneutrality Breakdown and Specific Ion Effects in Nanoconfined Aqueous Electrolytes Observed by NMR. Nat. Commun. 2015, 6, No. 6358 .

(14) Luo, Z.-X.; Xing, Y.-Z.; Liu, S.; Ling, Y.-C.; Kleinhammes, A.; $\mathrm{Wu}, \mathrm{Y}$. Dehydration of Ions in Voltage-Gated Carbon Nanopores Observed by In Situ NMR. J. Phys. Chem. Lett. 2015, 6, 5022-5026.

(15) Forse, A. C.; Griffin, J. M.; Merlet, C.; Bayley, P. M.; Wang, H.; Simon, P.; Grey, C. P. NMR Study of Ion Dynamics and Charge Storage in Ionic Liquid Supercapacitors. J. Am. Chem. Soc. 2015, 137, $7231-7242$

(16) Grey, C. P.; Tarascon, J. Sustainability and in situ Monitoring in Battery Development. Nat. Mater. 2017, 16, 45-56.

(17) Fedorov, M. V.; Kornyshev, A. A. Ionic Liquids at Electrified Interfaces. Chem. Rev. 2014, 114, 2978-3036.

(18) Hayes, R.; Warr, G. G.; Atkin, R. Structure and Nanostructure in Ionic Liquids. Chem. Rev. 2015, 115, 6357-6426.

(19) Zhang, S.; Zhang, J.; Zhang, Y.; Deng, Y. Nanoconfined Ionic Liquids. Chem. Rev. 2017, 117, 6755-6833.

(20) Smith, E. F.; Garcia, I. J. V.; Briggs, D.; Licence, P. Ionic Liquids in Vacuo; Solution-Phase X-ray Photoelectron Spectroscopy. Chem. Commun. 2005, 5633-5635.

(21) Smith, E. F.; Rutten, F. J.; Villar-Garcia, I. J.; Briggs, D.; Licence, P. Ionic Liquids in Vacuo: Analysis of Liquid Surfaces Using UltraHigh-Vacuum Techniques. Langmuir 2006, 22, 9386-9392.

(22) Taylor, A. W.; Qiu, F.; Villar-Garcia, I. J.; Licence, P. Spectroelectrochemistry at Ultrahigh Vacuum: in situ Monitoring of Electrochemically Generated Species by X-ray Photoelectron Spectroscopy. Chem. Commun. 2009, 5817-5819.

(23) Lovelock, K. R.; Villar-Garcia, I. J.; Maier, F.; Steinrück, H.-P.; Licence, P. Photoelectron Spectroscopy of Ionic Liquid-Based Interfaces. Chem. Rev. 2010, 110, 5158-5190.

(24) Qiu, F.; Taylor, A. W.; Men, S.; Villar-Garcia, I. J.; Licence, P. An Ultra High Vacuum-Spectroelectrochemical Study of the Dissolution of Copper in the Ionic Liquid (N-methylacetate)-4picolinium Bis (Trifluoromethylsulfonyl) Imide. Phys. Chem. Chem. Phys. 2010, 12, 1982-1990.

(25) Villar-Garcia, I. J.; Smith, E. F.; Taylor, A. W.; Qiu, F.; Lovelock, K. R.; Jones, R. G.; Licence, P. Charging of Ionic Liquid Surfaces Under X-ray Irradiation: The Measurement of Absolute Binding Energies by XPS. Phys. Chem. Chem. Phys. 2011, 13, 2797-2808.

(26) Weingarth, D.; Foelske-Schmitz, A.; Wokaun, A.; Kötz, R. In Situ Electrochemical XPS Study of the Pt/[EMIM][BF 4] System. Electrochem. Commun. 2011, 13, 619-622.

(27) Kolbeck, C.; Niedermaier, I.; Deyko, A.; Lovelock, K. R.; Taccardi, N.; Wei, W.; Wasserscheid, P.; Maier, F.; Steinrück, H. P. Influence of Substituents and Functional Groups on the Surface Composition of Ionic Liquids. Chem. - Eur. J. 2014, 20, 3954-3965.

(28) Matsuda, T.; Taccardi, N.; Schwegler, J.; Wasserscheid, P.; Steinrück, H. P.; Maier, F. Vacuum Surface Science Meets Heterogeneous Catalysis: Dehydrogenation of a Liquid Organic Hydrogen Carrier in the Liquid State. Chem. Phys. Chem. 2015, 16, 1873-1879.

(29) Camci, M. T.; Aydogan, P.; Ulgut, B.; Kocabas, C.; Suzer, S. XPS Enables Visualization of Electrode Potential Screening in an Ionic Liquid Medium with Temporal-and Lateral-Resolution. Phys. Chem. Chem. Phys. 2016, 18, 28434-28440.

(30) Camci, M. T.; Ulgut, B.; Kocabas, C.; Suzer, S. In-Situ XPS Monitoring and Characterization of Electrochemically Prepared Au Nanoparticles in an Ionic Liquid. ACS Omega 2017, 2, 478-486. 
(31) Polat, E. O.; Uzlu, H. B.; Balci, O.; Kakenov, N.; Kovalska, E.; Kocabas, C. Graphene-Enabled Optoelectronics on Paper. ACS Photonics 2016, 3, 964-971.

(32) Kakenov, N.; Balci, O.; Takan, T.; Ozkan, V. A.; Altan, H.; Kocabas, C. Observation of Gate-Tunable Coherent Perfect Absorption of Terahertz Radiation in Graphene. ACS Photonics 2016, 3, 1531-1535.

(33) Polat, E. O.; Balc1, O.; Kocabas, C. Graphene Based Flexible Electrochromic Devices. Sci. Rep. 2014, 4, No. 6484.

(34) Aygar, A. M.; Balci, O.; Cakmakyapan, S.; Kocabas, C.; Caglayan, H.; Ozbay, E. Comparison of Back and Top Gating Schemes with Tunable Graphene Fractal Metasurfaces. ACS Photonics 2016, 3, 2303-2307.

(35) Cohen, H. Chemically Resolved Electrical Measurements in Organic Self-Assembled Molecular Layers. J. Electron Spectrosc. Relat. Phenom. 2010, 176, 24-34.

(36) Lichterman, M. F.; Hu, S.; Richter, M. H.; Crumlin, E. J.; Axnanda, S.; Favaro, M.; Drisdell, W.; Hussain, Z.; Mayer, T.; Brunschwig, B. S.; et al. Direct Observation of the Energetics at a Semiconductor/Liquid Junction by Operando X-ray Photoelectron Spectroscopy. Energy Environ. Sci. 2015, 8, 2409-2416.

(37) Foelske-Schmitz, A.; Weingarth, D.; Kötz, R. Quasi in situ XPS Study of Electrochemical Oxidation and Reduction of Highly Oriented Pyrolytic Graphite in [1-ethyl-3-methylimidazolium][BF 4] Electrolytes. Electrochim. Acta 2011, 56, 10321-10331.

(38) Wibowo, R.; Aldous, L.; Jacobs, R. M.; Manan, N. S.; Compton, R. G. Monitoring Potassium Metal Electrodeposition From an Ionic Liquid Using in situ Electrochemical-X-ray Photoelectron Spectroscopy. Chem. Phys. Lett. 2011, 509, 72-76.

(39) Kruusma, J.; Tõnisoo, A.; Pärna, R.; Nõmmiste, E.; Tallo, I.; Romann, T.; Lust, E. Influence of the Negative Potential of Molybdenum Carbide Derived Carbon Electrode on the in situ Synchrotron Radiation Activated X-ray Photoelectron Spectra of 1ethyl-3-methylimidazolium Tetrafluoroborate. Electrochim. Acta 2016, 206, 419-426.

(40) Gokturk, P. A.; Salzner, U.; Nyulászi, L.; Ulgut, B.; Kocabas, C.; Suzer, S. XPS-Evidence for in-situ Electrochemically-Generated Carbene Formation. Electrochim. Acta 2017, 234, 37-42.

(41) Gokturk, P. A.; Donmez, S.; Ulgut, B.; Türkmen, Y.; Suzer, S. Optical and XPS Evidence for the Electrochemical Generation of an $\mathrm{N}$-heterocyclic Carbene and its CS 2 Adduct from the Ionic Liquid [Bmim][PF 6]. New J. Chem. 2017, 41, 10299-10304.

(42) Olschewski, M.; Gustus, R.; Höfft, O.; Lahiri, A.; Endres, F. Monochromatic X-ray Photoelectron Spectroscopy Study of Three Different Ionic Liquids in Interaction with Lithium-Decorated Copper Surfaces. J. Phys. Chem. C 2017, 121, 2675-2682.

(43) Södergren, S.; Siegbahn, H.; Rensmo, H.; Lindström, H.; Hagfeldt, A.; Lindquist, S.-E. Lithium Intercalation in Nanoporous Anatase TiO2 Studied with XPS. J. Phys. Chem. B 1997, 101, 30873090.

(44) Thißen, A.; Ensling, D.; Liberatore, M.; Wu, Q.-H.; Madrigal, F. F.; Bhuvaneswari, M.; Hunger, R.; Jaegermann, W. Experimental Routes to in situ Characterization of the Electronic Structure and Chemical Composition of Cathode Materials for Lithium Ion Batteries During Lithium Intercalation and Deintercalation Using Photoelectron Spectroscopy and Related Techniques. Ionics 2009, 15, 393-403.

(45) Foelske-Schmitz, A.; Ruch, P. W.; Kötz, R. Ion Intercalation into HOPG in Supercapacitor Electrolyte-An X-ray Photoelectron Spectroscopy Study. J. Electron Spectrosc. Relat. Phenom. 2010, 182, 57-62. (46) Schnyder, B.; Alliata, D.; Kotz, R.; Siegenthaler, H. Electrochemical Intercalation of Perchlorate Ions in HOPG: an SFM/LFM and XPS Study. Appl. Surf. Sci. 2001, 173, 221-232.

(47) Foelske-Schmitz, A.; Weingarth, D.; Kaiser, H.; Kötz, R. Quasi in situ XPS Study of Anion Intercalation into HOPG From the Ionic Liquid [EMIM][BF4]. Electrochem. Commun. 2010, 12, 1453-1456.

(48) Radhakrishnan, G.; Cardema, J. D.; Adams, P. M.; Kim, H. I.; Foran, B. Fabrication and Electrochemical Characterization of Single and Multi-Layer Graphene Anodes for Lithium-Ion Batteries. J. Electrochem. Soc. 2012, 159, A752.
(49) Zhang, C.; Grass, M. E.; Yu, Y.; Gaskell, K. J.; DeCaluwe, S. C.; Chang, R.; Jackson, G. S.; Hussain, Z.; Bluhm, H.; Eichhorn, B. W.; et al. Multielement Activity Mapping and Potential Mapping in Solid Oxide Electrochemical Cells Through the use of Operando XPS. ACS Catal. 2012, 2, 2297-2304.

(50) Maier, F.; Gottfried, J. M.; Rossa, J.; Gerhard, D.; Schulz, P. S.; Schwieger, W.; Wasserscheid, P.; Steinrück, H. P. Surface Enrichment and Depletion Effects of Ions Dissolved in an Ionic Liquid: An X-ray Photoelectron Spectroscopy Study. Angew. Chem., Int. Ed. 2006, 45, $7778-7780$.

(51) Lockett, V.; Sedev, R.; Bassell, C.; Ralston, J. Angle-Resolved Xray Photoelectron Spectroscopy of the Surface of Imidazolium Ionic Liquids. Phys. Chem. Chem. Phys. 2008, 10, 1330-1335.

(52) Maier, F.; Cremer, T.; Kolbeck, C.; Lovelock, K.; Paape, N.; Schulz, P.; Wasserscheid, P.; Steinrück, H.-P. Insights Into the Surface Composition and Enrichment Effects of Ionic Liquids and Ionic Liquid Mixtures. Phys. Chem. Chem. Phys. 2010, 12, 1905-1915.

(53) Olschewski, M.; Gustus, R.; Marschewski, M.; Höfft, O.; Endres, F. Spectroscopic Characterization of the Interaction of Lithium with Thin Films of the Ionic Liquid 1-octyl-3-methylimidazolium bis (trifluoromethylsulfonyl) amide. Phys. Chem. Chem. Phys. 2014, 16, 25969-25977.

(54) Copuroglu, M.; Aydogan, P.; Polat, E. O.; Kocabas, C.; Süzer, S. Gate-Tunable Photoemission from Graphene Transistors. Nano Lett. 2014, 14, 2837-2842.

(55) Fujimoto, T.; Awaga, K. Electric-Double-Layer Field-Effect Transistors with Ionic Liquids. Phys. Chem. Chem. Phys. 2013, 15, 8983-9006.

(56) Brown, M. A.; Redondo, A. B.; Sterrer, M.; Winter, B.; Pacchioni, G.; Abbas, Z.; van Bokhoven, J. A. Measure of Surface Potential at the Aqueous-Oxide Nanoparticle Interface by XPS from a Liquid Microjet. Nano Lett. 2013, 13, 5403-5407.

(57) Brown, M. A.; Abbas, Z.; Kleibert, A.; Green, R. G.; Goel, A.; May, S.; Squires, T. M. Determination of Surface Potential and Electrical Double-Layer Structure at the Aqueous Electrolyte-Nanoparticle Interface. Phys. Rev. X 2016, 6, No. 011007.

(58) Huang, M. M.; Jiang, Y.; Sasisanker, P.; Driver, G. W.; Weingartner, H. Static Relative Dielectric Permittivities of Ionic Liquids at $25^{\circ} \mathrm{C}$. J. Chem. Eng. Data 2011, 56, 1494-1499.

(59) MacFarlane, D. R.; Forsyth, M.; Izgorodina, E. I.; Abbott, A. P.; Annat, G.; Fraser, K. On the Concept of Ionicity inIonic Liquids. Phys. Chem. Chem. Phys. 2009, 11, 4962-4967. 